\title{
When prevention is a risk. More is not always better
}

\author{
Sergio Baratta \\ Non-Invasive Cardiology, Hospital Universitario Austral, Buenos Aires, Argentina
}

\begin{abstract}
Article p. 510
In spite of complex interactions between drugs and dietary supplements, which increase with the number of them taken, the medical, or on occasions, the patient's judgment tends to be fast and dichotomous. Many contexts of clinical practice do not have scientific evidence derived from randomized clinical trials and action is based on physiopathological or simply epidemiological analysis, and in worst cases personal or common beliefs.
\end{abstract}

Karny-Rahkovich et al's. study [1] evaluated the use of dietary supplements in 149 patients with cardiovascular (CV) disease who consulted an Internal Medicine Ward and an Emergency Cardiology Clinic. Despite being a small sample, high use of dietary supplements (45\%) associated with older age, female sex and a physical activity routine was observed. They also identified 16 potential interactions between the prescribed medical treatment and dietary supplements. It would have been interesting to compare the rate of use of dietary supplements with a control group without $\mathrm{CV}$ disease. The study repeats the finding that people who carry out physical activity refer greater consumption of potentially harmful supplements of unproven usefulness. The work details the potentially dangerous interactions between drugs and dietary supplements, although the clinical impact is not fully known [1].

In this sense, the association of elevated levels of homocysteine with higher CV risk and the possibility of its descent through the use of folic acid lead many our colleagues to indicate it systematically. The subsequent randomized studies have shown that the administration of folic acid to reduce levels of homocysteine not only does not reduce CV risk, they also showed that the administration of vitamin $B$ caused an increase in mortality [2].

The association between vitamin $\mathrm{E}$ intake and lower incidence of $\mathrm{CV}$ events was demonstrated following an epidemiological prospective observational study conducted on nurses. This finding generated a large increase in the consumption of antioxidants such as vitamin E, even among American doctors. Subsequently, several randomized clinical trials on large cohorts highlighted the absence of any protective effect on vascular events and even a higher risk if used in high doses [3].

In spite of the benefits of vitamin $\mathrm{C}$ on endothelial function and the popular acceptance of its benefits, the systematic administration of a vitamin complex (600 mg vitamin E, $250 \mathrm{mg}$ vitamin C, and $20 \mathrm{mg}$ beta-carotene daily) in a multicenter study dismissed the improvement in the prognosis, yet not causing higher rate of adverse effects [4].

Finally, a meta-analysis that included 68 studies showed that the administration of antioxidants was associated with an increase in mortality: beta-carotene (vitamin B) - RR 1.07 (95\% CI 1.02-1.11), vitamin A - RR 1.16 (95\% CI 1.1-1.24), vitamin E - RR 1.04 (95\% CI 1.01-1.07) with no defined adverse effects and no clear benefits with vitamin $\mathrm{C}$ or selenium supplements [5].

The evidence showing CV benefit from the use of vitamin D is not conclusive in spite of the fact that in epidemiological studies the reduction in vitamin D levels has been linked to an increased risk of developing CV disease. Vitamin D3 seems to decrease the mortality in the elderly, vitamin D2, alfacalcidol and calcitriol do not have statistically significant effects on mortality, vitamin D3 combined with calcium increases the rate of nephrolithiasis and alfacalcidol as well as calcitriol

Address for correspondence: Dr Sergio Baratta, Chief, Non Invasive Cardiology, Hospital Universitario Austral, Av. Juan Domingo Perón 1500 (B1629AHJ), Pilar, Buenos Aires, Argentina, e-mail: sjbaratta@yahoo.com.ar; sbaratta@cas.austral.edu.ar

Received: 05.07.2015 Accepted: 06.07.2015 
increase hypercalcemia. Given the biases of the published studies, the dropout of patients included in the report of mortality, and the weakness of the available evidence, large cohort randomized studies are needed to compare the administration of vitamin D vs. placebo [6].

Despite the use of dietary supplements, there are no studies demonstrating their use in the primary prevention of $\mathrm{CV}$ disease or cancer nor is there enough information to analyze the clinical impact of their individual interaction in daily practice [7].

The available evidence establishes that the utility of dietary supplements is part of the belief and expectations of the patient and is sometimes a desire of the pharmaceutical industry. Given that the clinical benefits have not been tested, it is best not to prescribe them, avoid erroneous medical practices and in the best of cases to encourage a deeper discussion in the community and a more cautious approach in doctors and patients.

Conflict of interest: None declared

\section{References}

1. Karny-Rahkovich O, Blatt A, Elbaz-Greener G et al. Dietary supplement consumption among cardiac patients admitted to internal medicine and cardiac wards. Cardiol J, 2015; 22: 510-518.

2. Bønaa KH, Njølstad I, Ueland PM et al. Homocysteine lowering and cardiovascular events after acute myocardial infarction. N Engl J Med, 2006; 354: 1578-1588.

3. Miller ER 3rd, Pastor-Barriuso R, Dalal D et al. Meta-analysis: high-dosage vitamin $\mathrm{E}$ supplementation may increase all-cause mortality. Ann Intern Med, 2005; 142: 37-46.

4. Heart Protection Study Collaborative Group. MRC/BHF Heart Protection Study of antioxidant vitamin supplementation in 20,536 high-risk individuals: a randomized placebo-controlled trial. Lancet, 2002; 360: 23-33.

5. Bjelakovic G, Nikolova D, Gluud LL et al. Mortality in randomized trials of antioxidant supplements for primary and secondary prevention: systematic review and meta-analysis. JAMA, 2007; 297: 842-857.

6. Bjelakovic G, Gluud LL, Nikolova D et al. Vitamin D supplementation for prevention of mortality in adults. Cochrane Database Syst Rev, 2014; 1: CD007470.

7. Fortmann SP, Burda BU, Senger CA et al. Vitamin, mineral, and multivitamin supplements for the primary prevention of cardiovascular disease and cancer: A Systematic Evidence Review for the U.S. Preventive Services Task Force [Internet]. Rockville (MD): Agency for Healthcare Research and Quality (US); 2013 Nov. Available from http:/www.ncbi.nlm.nih.gov/books/ NBK173987/. 